\title{
A Variable Bandwidth Memristor-Based Legendre Optimum Low-Pass Filter for Radio Frequency (RF) Applications
}

\author{
Elochukwu Onyejegbu ${ }^{1}$, Zhazira zhumsbay $^{1}$, Arjuna Marzuki², and Ikechi Ukaegbu ${ }^{1}$ \\ ${ }^{1}$ Nazarbaev universitet \\ ${ }^{2}$ Wawasan Open University
}

February 14, 2022

\begin{abstract}
This paper presents the enhancement of Legendre Optimum low pass filters in terms of reusability and bandwidth, based on the variable or programmable memristance of memristors. Two low pass filters, of third and fifth order, operating in the radio frequency range, and designed using the insertion loss method are presented. At $600 \mathrm{KHz}$ and at $110 \mathrm{MHz}$, two MS memristor models, of the non-linear ion drift class is incorporated into the filter circuits in turn and their memristances varied such that R_off- R_on decreases monotonically and R_off- R_on>0. Results show a bandwidth enhancement of up to $100 \mathrm{KHz}$ at 600 $\mathrm{KHz}$, and up to $19 \mathrm{MHz}$ at $110 \mathrm{MHz}$. This study also examines the effect of the simultaneous versus asynchronous variation of the memristance of the pair of memristors introduced into the filter circuits, as well as increase in filter order.
\end{abstract}

\section{Hosted file}

A Variable Bandwidth Memristor-Based Legendre Optimum Low-Pass Filter for RF Applications.pdf available at https://authorea.com/users/460278/articles/556321-a-variable-bandwidthmemristor-based-legendre-optimum-low-pass-filter-for-radio-frequency-rf-applications 